be important access points for syphilis and drug use prevention.

Disclosure No significant relationships.

\section{P768 THE EVOLUTION OF AN INFECTIOUS SYPHILIS EPIDEMIC IN A CANADIAN URBAN SETTING}

Souradet Shaw*, Alicia Lapple, Joss Reimer, Craig Ross, Debbie Nowicki, Lawrence Elliott, Pierre Plourde. Winnipeg Regional Health Authority, Winnipeg, Canada

10.1136/sextrans-2019-sti.826

Background Reflecting worldwide trends, an outbreak of infectious syphilis was declared in Winnipeg, Canada in 2012. Initially exclusively an outbreak amongst men who have sex with men (MSM), increases among women were observed in 2014/ 15. This study compared the characteristics of more recent heterosexual cases of syphilis to those at the start of the heterosexual outbreak.

Methods Data were from infectious syphilis investigations (September 1st, 2011 to August 31st, 2018) from Winnipeg, Canada. Age-standardized rates (2006 Canadian population standard) and 95\% confidence intervals $(95 \% \mathrm{CI})$ are reported. Bivariate analyses ( $\chi^{2}$ tests) and multivariable logistic regression models compared heterosexual cases from 2011/12-2014/ 15 to 2015/16-2017/18 cases on socio-demographic characteristics and risk factors. Adjusted odds ratios (AOR) and 95\% CI are reported.

Results A total of 770 infectious syphilis cases were reported to public health. Rates increased 25 -fold, from 1.6/100,000 persons (95\%CI:0.8-2.9) to $40.5 / 100,000$ persons (95\%CI: $36.1-45.3$ ), between $2011 / 12$ and $2017 / 18$. The proportion of women increased from $8 \%$ to $47 \%$; correspondingly, the ratio between male and female rates decreased from 4.5 to 1.3 . Amongst men, the heterosexual proportion increased from $9 \%$ in $2011 / 12$ to $75 \%$ in $2017 / 18$. In bivariate analyses, more recent heterosexual cases were younger $(\mathrm{p}<0.001)$; more likely to report crystal methamphetamine (CM) use $(30 \%$ vs $3 \%$, $\mathrm{p}<0.001)$; gonorrhea $(21 \%$ vs $3 \%, \mathrm{p}<0.001)$ and chlamydia co-infection $(23 \%$ vs $10 \%, \mathrm{p}=0.004)$; history of incarceration (36\% vs $14 \%, \mathrm{p}<0.001)$; and having no fixed address $(15 \%$ vs $2 \%, \mathrm{p}<0.001)$. In multivariable models, CM use (AOR: 7.9; 95\%CI:2.4-26.7), gonorrhea co-infection (AOR: 7.1; 95\%CI: 2.1-24.1), and history of incarceration (AOR: 1.8; 95\%CI: 1.1-3.5) remained statistically significant.

Conclusion At least two parallel epidemics of syphilis are occurring in Winnipeg. Although MSM cases have declined, control of the heterosexual outbreak remains elusive. Populations with multiple vulnerabilities, including CM use and history of incarceration bear the greatest burden. Public health surveillance should remain vigilant for congenital syphilis.

Disclosure No significant relationships.

\section{P769 A SYSTEMATIC REVIEW ON ALTERNATIVE TREATMENTS FOR MATERNAL SYPHILIS}

Aishwarya Raich*, Chelsea Roberts, Chrysovalantis Stafylis, Jeffrey Klausner. UCLA David Geffen School of Medicine, Infectious Diseases, Los Angeles, USA

10.1136/sextrans-2019-sti.827

Background Maternal syphilis leads to preventable adverse fetal health outcomes. The recommended treatment is benzathine penicillin, which is challenging due to shortages, drug allergies and inability to administer the injection. We conducted a literature review to identify potential treatment options for maternal syphilis.

Methods We searched PubMed, Embase, and Scopus from January 1,1970 to December 31, 2018. The search terms "syphilis" AND ("maternal” OR "pregnancy" OR "congenital”), AND "treatment" NOT ("screening") were used. Additional articles were identified from the references. We included studies in English, with full text, on humans and women. Successful treatment was defined as maternal RPR titer decline and congenital syphilis prevention.

Results Of the 70 articles, 8 case series were included. 11 pregnant women were successfully treated with intramuscular ceftriaxone $250 \mathrm{mg}$ : 7-day course for primary syphilis or 10day course for secondary syphilis, repeated at 28-weeks gestation. One patient was successfully treated with amoxicillin $6 \mathrm{~g}$ and probenecid $1 \mathrm{~g}$ daily for 14 days, and another was successfully treated with a 6-day course of amoxicillin followed by ceftriaxone $2 \mathrm{~g}$ intravenously for 8 days. In response to ampicillin $2 \mathrm{~g}$ intravenously intrapartum, one mother and one neonate developed the Jarish-Herxheimer reaction in cases of undiagnosed syphilis. Macrolides failed to prevent congenital syphilis: 5 patients treated with azithromycin $1 \mathrm{~g}$ orally for 110 days, one case report of erythromycin $750 \mathrm{mg}$ orally QID for 12 days and one case report of two failed 15-day courses of oral erythromycin 750-800 mg QID until penicillin desensitization was initiated. One case of clindamycin decreased maternal RPR titers, but failed to prevent congenital syphilis. Conclusion Overall, 23 patients were treated with penicillin alternatives (15 with beta-lactam antibiotics, 7 with macrolide antibiotics, 1 with clindamycin), and 13 were treated successfully. Clinical research should evaluate amoxicillin and cephalosporins. Our review does not support the use of macrolide antibiotics.

Disclosure No significant relationships.

\section{P770 JARISCH-HERXHEIMER REACTION IN CENTRAL NERVOUS SYSTEM AMONG NEUROSYPHILIS PATIENTS: DISCONTINUATION OF THERAPY OR NOT?}

Rui-Rui Peng*, Juan Wu, Wei Zhao, Lin Zhu, Sheng Lu, Xin Gu, Zhi-Fang Guan, Pingyu Zhou. Sexually Transmitted Disease Institute, Shanghai Skin Disease Hospital, Shanghai, China

\subsection{6/sextrans-2019-sti.828}

Background Syphilis has returned to china with a vengeance since the 21st century, and the epidemiology of neurosyphilis has largely paralleled that of active syphilis. Prompt therapy with high-dose intravenous benzylpenicillin is critical to alleviate clinical symptoms of neurosyphilis patients. However, patients may experience an exacerbation of mental and/or neurological symptoms following the initiation of treatment due to a severe Jarisch-Herxheimer reaction (JHR) in central nervous system (CNS). We retrospectively analyzed the incidence, risk factors and prognosis for JHR in CNS in Shanghai Skin Disease Hospital, China.

Methods From July 1, 2017 to December 31, 2018 at our sexually transmitted disease ward, 574 neurosyphilis patients received the high-dose intravenous benzylpenicillin. Patient factors were recorded, including age, gender, neurosyphilis type, serum and cerebrospinal fluid-venereal disease research laboratory test (CSF-VDRL) titer, white blood cell count and protein 